\title{
The Motion Estimation Research Based on the Histogram of Canny Edge Feature Points
}

\author{
Q. Zhao \\ Xi'an University of Science and Technology, School of \\ Communication and Information Engineering
} Xi’an7, China

Y.X. Jia

Xi'an University of Science and Technology, School of Communication and Information Engineering Xi'an, China

$$
\text { S. Zhao }
$$

Xi'an Municipal Facilities Administration Bureau Xi'an , China

\begin{abstract}
The criteria of traditional block matching motion estimation algorithm is based on the gray value offset between macro block, which was sensitive to the noise and the treatment under uneven illumination environment. For above problem, the paper proposed that using canny algorithm extracted the edge profile of current frame, and then calculate the feature points distribution histogram of block estimated, finally, using the the distance of feature points histogram between two frames as matching criteria for all frames. The experiment showed that, the motion estimation accuracy of improved algorithm is high, and it has small difference between target and matching box. Improved algorithm has better robustness on the scene affected by the noise and poor illumination, and it can apply to intelligent image processing work of coal mine and other special environments.
\end{abstract}

Keywords-canny; motion estimation; edge detection; histogram matching

\section{INTROUCTION}

The purpose of motion estimation is to find out the moving relationship between consecutive frames, playing an important role in video coding, data compression, video tracking and other fields. The traditional motion estimation method mainly include pixel iteration method, the global motion estimation method, fast matching method, etc. The block matching method because of its simple and easy to implement, and is widely used. Traditional block matching method has the full search method, the three-step search (TSS) method, four-step search method, diamond search (DS), block-based gradient descent search (BBGDS), hexagonal method (HEXBS)[1-2], and so on. The ideas of these algorithms are all based on a certain size divide the reference image into a number of macro blocks, and then searching for the matching sub-block in the next frame according to the specific matching criterion[3-4]. Commonly used matching criterion have mean absolute error (MAD),

\author{
Y.B. Hou \\ Xi'an University of Science and Technology, School of \\ Electrical and Control Engineering \\ Xi'an, China
}

S.W. Kou

Xi'an University of Science and Technology, School of Communication and Information Engineering

Xi'an, China

X.R. Mao

Xi'an University of Science and Technology, School of Communication and Information Engineering

Xi'an, China

mean square error (MSE) and normalized cross-correlation function(NFFC),since MAD has no multiplication and division operation, do not need to do multiplication, and which is simple and convenient, so used more. Usually use the sum of absolute error (SAD) instead of MAD.

Because these useful matching criterias are based on the comparison of gray value difference between the macroblocks, in the motion estimation, which is very sensitive to the changes of light and shade of image and noise, and comparing with grey value cannot reflect the distribution patterns of image. Therefore this article consider that to get the contours of the image at the first and to find out the feature points distribution histogram of specified macro blocks in the reference frame, and then using diamond method to search 2nd frame, finding out the smallest distance macro block with the specified macro block as the best matching block.

\section{ALGORITHM INTRODUCTION}

\section{A. DS method}

DS method is also called diamond method, which is simple, robust, and efficient and so on, and which is a fast search algorithm. This algorithm is similar with four-step method, making two patterns according to the desired shape and size of the search, large diamond search pattern (LDSP) and small diamond search pattern (SDSP). Among them, large diamond search pattern has 9 points to be searched, and small diamond search pattern has 5 points to be searched.

Searching process of DS method is as follows:

1). Making the center coordinates of macro block to be estimated in the reference as the center coordinates of 2nd image LDSP pattern, and get the matching error of the center point and around the 8 points; 
2). If the minimum matching error in around eight points, and then called the point as a new center, and then back to the first step to calculate, otherwise, the third step;

3). If the minimum matching error in the center point, then the point as the center point of SDSP pattern, and get the minimum error on the pattern and setting as the final center point of matching macro block.

The example of DS method is as fellow figure:

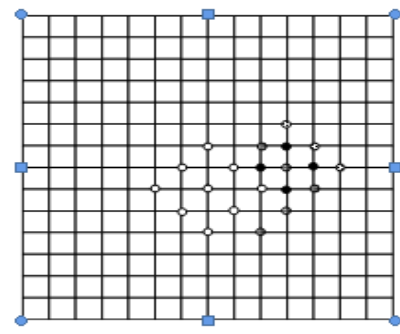
: plate selected in $1^{\text {st }}$ step
: plate selected in $2^{\text {nd }}$ step
(1) : plate selected in $3^{\text {rd }}$ step
: plate selected in $4^{\text {th }}$ step
FIGURE I. SEARCHING PROCESS OF DS METHOD

\section{B. Canny operator}

Canny operator [5-6] is a commonly used edge detection operator, which has high positioning performance, and has a better signal-to-noise ratio. Canny algorithm steps as follows:

1). Using Gaussian filter to the image, effectively removing the noise of the image, while having a smoothing effect;

2). Using first order partial finite difference and getting gradient magnitude and direction of each pixel in the image.

$$
\left\{\begin{array}{l}
E_{x}[x, y]=(T(i+1, j)-T(i, j)+T(i+1, j+1)-T(i+1, j)) / 2 \\
E_{y}[x, y]=(T(i, j)-T(i+1, j)+T(i, j+1)-T(i+1, j+1)) / 2
\end{array}\right.
$$

The gradient amplitude of each point $A(i, j)$ in the image $f(x, y)$;

$$
A(i, j)=\sqrt{E_{x}[i, j]^{2}+E_{y}[i, j]^{2}}
$$

Gradient direction of each point in the image $\theta(i, j)$ :

$$
\theta(i, j)=\arctan \left[E_{y}(i, j) / E_{x}(i, j)\right\rfloor
$$

3). Using the maximum inhibition for the obtained gradient values of pixel, that is, if gradient value of some element point is greater than the gradient vale of adjacent two element points on the gradient direction, then thought the point as edge point, setting to 1 , on the contrary, setting to 0 ;

4). Setting high and low thresholds, the gradient value between high and low threshold element thought as edge point, and then connecting all the edge points, forming the image outline.

\section{THE NEW ALGORITHM PROPOSED}

As mentioned above, in the traditional block matching algorithms, all matching criteria are based on the gray value deviation between macro block, therefore, which is very sensitive to the noise and change of light and shade for the outside. Firstly, canny operator uses Gaussian filter on the graph, so there is some noise immunity. Secondly, because what the matching is the outline of image, which is not sensitive to the change of the light and shade. This article consider using outline to do matching, it is required not too much for external conditions compared with using gray scale deviation to do matching.

These advantages based on canny algorithm, this article proposed using canny algorithm to get the outline of the image at the first, and setting the edge point to 1 , otherwise setting to 0 , then calculating the histogram of the number of the edge points in each column of each macro block. Finally, using the distance between the macro block is regarded as the new criterion.

Algorithm steps:

1). Using canny algorithm to get the outline of each frame, and setting the edge points value is 1 , otherwise, setting to 0 .

2). Determining the size of macro block, the row is $\mathrm{N}$, the column is $\mathrm{M}$, and calculate the feature points histogram of each macro block.

$$
\operatorname{imhist}(n)=\sum_{i=1}^{N} f\left(x-\frac{N}{2}+n, y-\frac{M}{2}+i\right) \quad n=1,2, \cdots N
$$

Where, $(x, y)$ is the coordinates of current matching point, $f$ is the macro block based on the center of $(x, y)$.

3). Using the distance between macro block as matching criterion, getting the original point $(x, y)$ in the second frame and the feature points distribution histogram of centered on 8 points surrounding the pattern of macro block. Finding the pixel of the smallest distance of macro block histogram with current pixel in the reference frame among 9 points, which is the new center. Iteration based on DS method, until finding the best matching point.

Matching criteria as follows: 


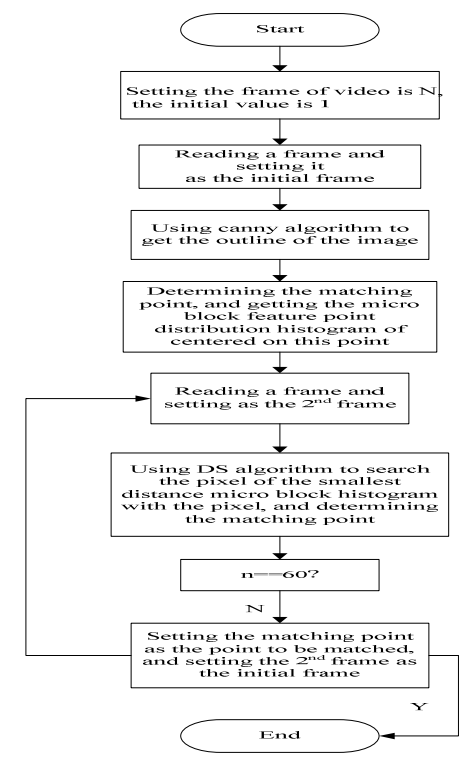

FIGURE II. THE FLOW CHART OF THE IMPROVED DS METHOD

$$
D\left(H_{1}, H_{2}\right)=\sum_{i=1}^{N}\left|H_{1}(i)-H_{2}(i)\right|
$$

Where, $\mathrm{H} 1$ is the macro block of current pixel, $\mathrm{H} 2$ is the macro block of each point on the pattern in the second frame, $\mathrm{N}$ is the column of macro block.

After using the canny algorithm to get the outline, because the edge point value is 1 , otherwise, the point value is 0 , the value of each column on each macro block histogram refers to each column contains the number of the edge points on macro block. Through the comparison of the feature points on two macro blocks, clearly distinguish the difference of the pixels on the two macro blocks. Figure 2 is the flow chart of improved algorithm.

\section{EXPERIMENT RESULT AND CONCLUSION}

This article based on Matlab 7.5, and chooses a period of video and doing the motion estimation simulation experiment with traditional DS method and this algorithm.

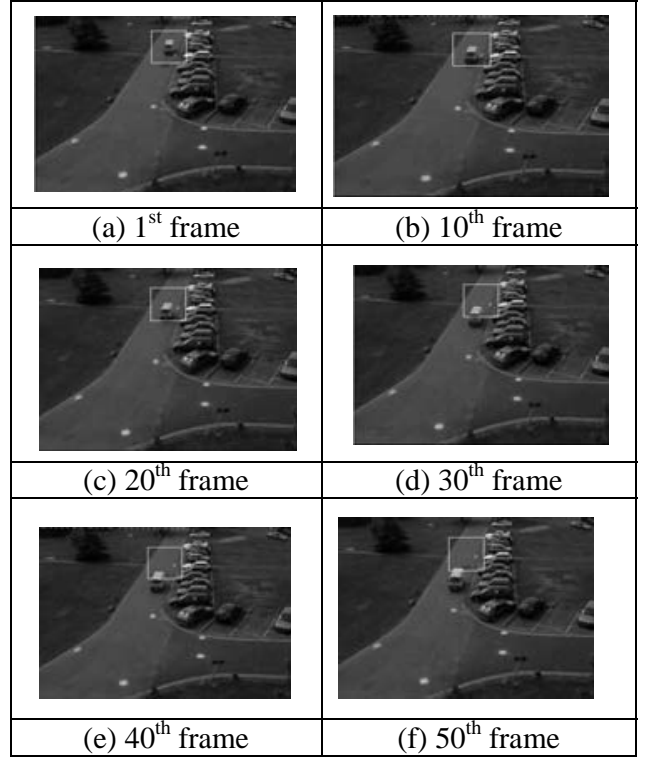

FIGURE III. Motion Estimation of Original DS Method

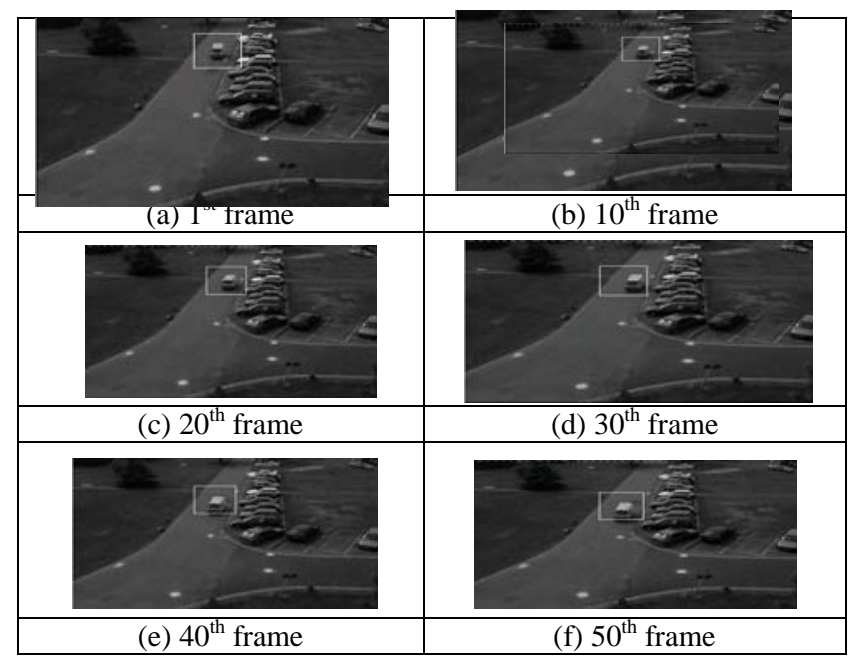

FIGURE IV. Motion Estimation of Improved DS Method

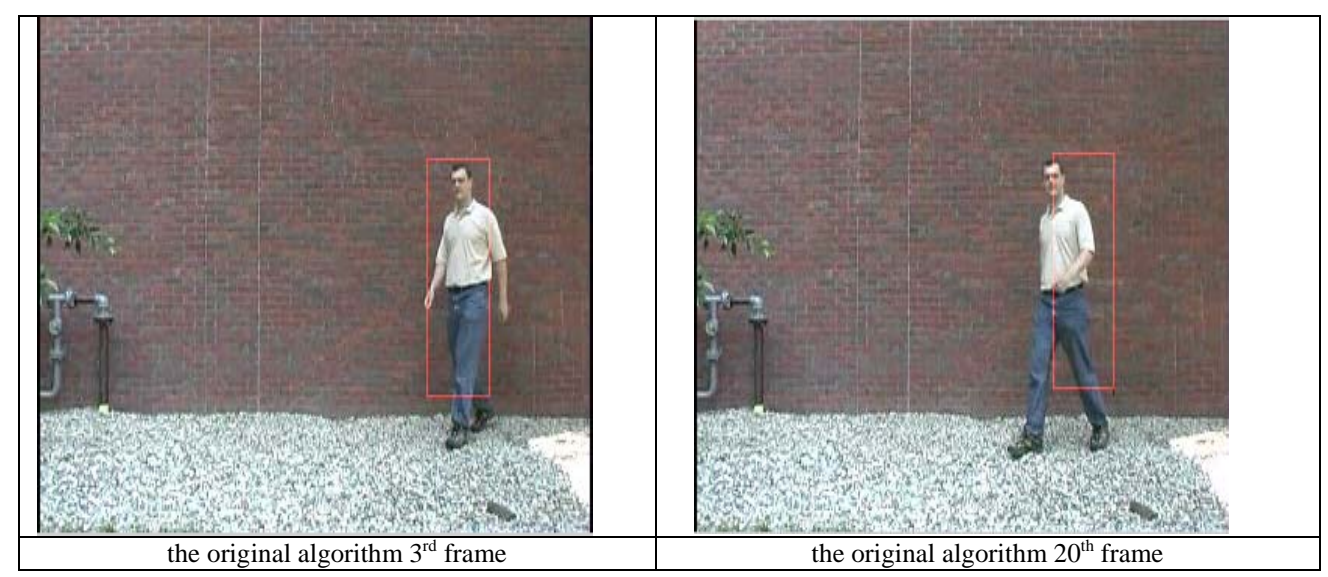

FIGURE V. MOTION ESTIMATION OF ORIGINAL DS METHOD 


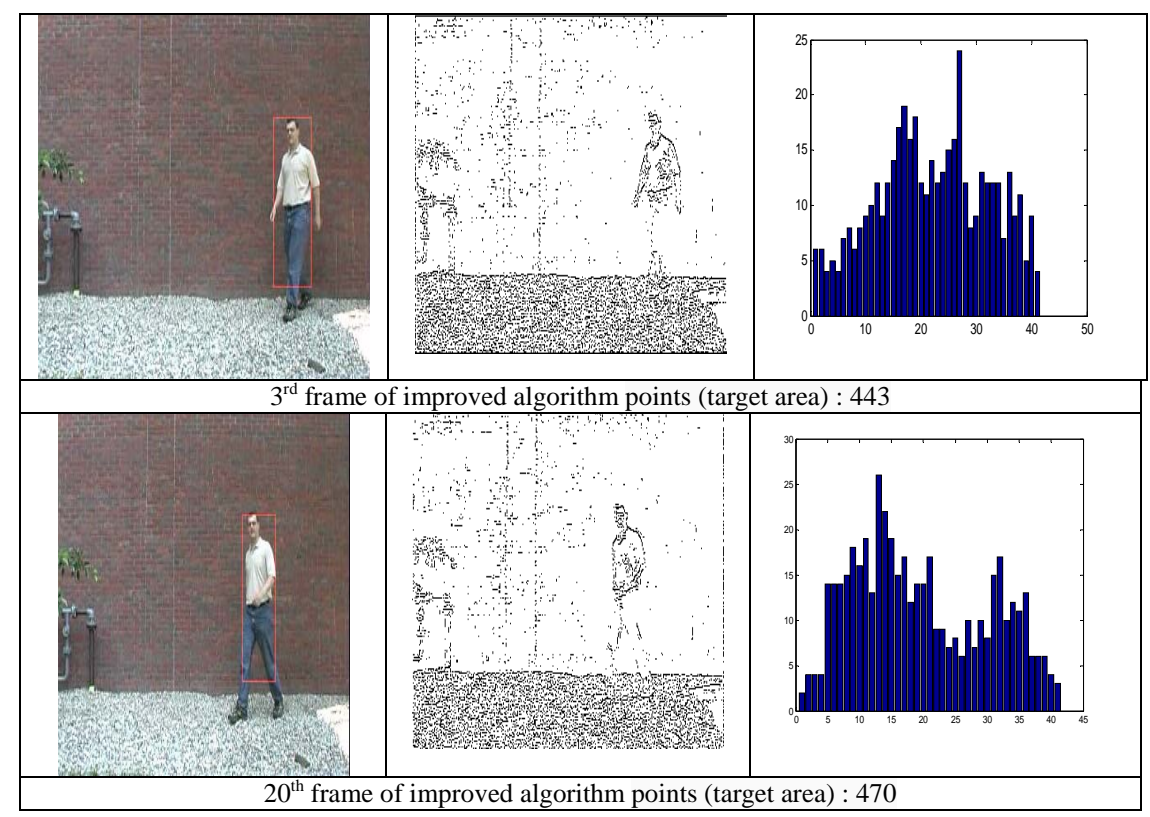

FIGURE VI. HISTOGRAM STATISTICS RESULTS OF IMPROVED DS METHOD.

Figure 3 is the motion estimation of original DS method, (a) is the 1st frame, that is the initial motion estimation of the image, (b) is the 10th frame estimation result, (c) is the 20th frame estimation result, (d) is the 30th frame estimation result, (e) is the 40th frame estimation result, (f) is the 50th frame estimation result.

Can be seen from figure 3 and figure 4, the pixel estimated by the improved DS method is much more accurate than traditional DS method. The latter lost the target in the 50th frame, but the former estimates the pixel basically accurate in the 50th frame.

\section{CONCLUSION}

This article proposed a traditional block matching of DS method, using the canny algorithm to get the outline point of the image at the first, determining the size of matching macro block and calculating the feature points distribution histogram of each macro block. Using histogram distance of macro block as matching criterion estimates motion. Experimental results show that the improved DS algorithm is much more accurate than the traditional DS algorithm.

\section{ACKNOWLEDGMENTS}

This work was supported in part by the Science and Technology Plan of Shaanxi Province, China (Program No. 2013K07-35; 2014K06-37), the Science and Technology Plan Industrial Application Technology Research and Development Project of Xi'an (Program No.CXY1343(4)), the Xi'an City Beilin District Application Technology Research and Development Project (Program No. GX1417; GX1310; GX1311).

\section{REFERENCE}

[1] XU Jic-bin, PO Lai-mao, CHEUNG Chok-kwan. Adaptive Motion Tracking Block Matching Algorithms for Video Coding [J].IEEE Trans on Circuits and System for Video Technology, 9(7):10251029, 1999.

[2] C.L.Jian , Ishfaq Ahmad and Z.L.Xi. An Adaptive Cross Search Algorithm for Block Matching Motion Estimation [J].IEEE Department of Computer Science and Engineering,5(2):914-918, 2004.

[3] SRINIVASAN R, RAO K R.Predictive Coding Based on Efficient Motion Estimation[J].IEEE Trans on Communications, COM 33:888-896,1985.

[4] C.Tan, C.Y.Ji, J.S.Gong, Y.D.Zhao J.H.Wei. Efficient algorithm of block matching, motion estimation based on Canny operator [J]. Journal of Harbin University of Commerce, 28(1):88-90, 2012.

[5] Y.Tang. The adaptive threshold of canny operator in the application of the image edge extraction [J].Silicon Valley (19): 132-141, 2011.

[6] Rafael C. Gonzalez. Digital Image Processing[M].Publishing House of Electronics Industry,2010. 